\title{
Exact solution for a fermion in the background of a scalar inversely linear potential
}

\author{
Antonio S. de Castro \\ UNESP - Campus de Guaratinguetá \\ Departamento de Física e Química \\ Caixa Postal 205 \\ 12516-410 Guaratinguetá SP - Brasil \\ E-mail address: castro@feg.unesp.br (A.S.de Castro)
}




\begin{abstract}
The problem of a fermion subject to a general scalar potential in a twodimensional world is mapped into a Sturm-Liouville problem for nonzero eigenenergies. The searching for possible bounded solutions is done in the circumstance of power-law potentials. The normalizable zero-eigenmode solutions are also searched. For the specific case of an inversely linear potential, which gives rise to an effective Kratzer potential, exact bounded solutions are found in closed form. The behaviour of the upper and lower components of the Dirac spinor is discussed in detail and some unusual results are revealed.
\end{abstract}


The Coulomb potential of a point electric charge in a $1+1$ dimension, considered as the time component of a Lorentz vector, is linear $(\sim|x|)$ and so it provides a constant electric field always pointing to, or from, the point charge. This problem is related to the confinement of fermions in the Schwinger and in the massive Schwinger models [1]-[2] and in the Thirring-Schwinger model 3]. It is frustrating that, due to the tunneling effect (Klein's paradox), there are no bound states for this kind of potential regardless of the strength of the potential [4]-[5]. The linear potential, considered as a Lorentz scalar, is also related to the quarkonium model in one-plus-one dimensions [6]- 7 . Recently it was incorrectly concluded that even in this case there is solely one bound state [8]. Later, the proper solutions for this last problem were found [9]-[1]. The mixed vector-scalar potential has also been analyzed for a linear potential [12 as well as for a general potential which goes to infinity as $|x| \rightarrow \infty$ [13. In both of those last references it has been concluded that there is confinement if the scalar coupling is of sufficient intensity compared to the vector coupling.

The problem of a particle subject to an inversely linear potential in one spatial dimension $\left(\sim|x|^{-1}\right)$, known as the one-dimensional hydrogen atom, has received considerable attention in the literature. This problem presents some conundrums and the most perplexing is that one regarding the ground state. The nonrelativistic Schrödinger equation provides a ground-state solution with infinite eigenenergy and a related eigenfunction given by a delta function centered about the origin. This problem was also analyzed with the Klein-Gordon equation and there it was revealed a finite eigenenergy and a exponentially decreasing eigenfunction [14. By using the technique of continuous dimensionality the problem was approached with the Schrödinger, Klein-Gordon and Dirac equations. The conclusion in this more recent work is that the Klein-Gordon equation provides unacceptable solutions while the Dirac equation, with the interacting potential considered as a time component of a vector, has no bounded solutions at all [15. This problem was also sketched for a Lorentz scalar interacting potential in the Dirac equation [16], but the analysis is incomplete.

In the present paper it is shown that the problem of a fermion under the influence of a general scalar potential for nonzero eigenenergies can be mapped into a Sturm-Liouville problem. Next the key conditions for the existence of bound-state solutions are settled for power-law potentials. The possible zero-mode solutions are shown to conform with the ultrarelativistic limit of the theory. In addition, the solution for an inversely linear potential 
is obtained in closed form. The effective potential resulting from the mapping has the form of the Kratzer potential [17. It is noticeable that this problem has an infinite number of acceptable bounded solutions, nevertheless it has no nonrelativistic limit for small quantum numbers. It is also shown that in the regime of strong coupling additional zero-energy solutions can be obtained as a limit case of nonzero-energy solutions. The ideas of supersymmetry have already been used to explore the two-dimensional Dirac equation with a scalar potential [18-19], nevertheless the power-law potential has been excluded of such discussions.

The two-dimensional Dirac equation can be obtained from the four-dimensional one with the mixture of spherically symmetric scalar, vector and anomalous magnetic-like (tensor) interactions. If we limit the fermion to move in the $x$-direction $\left(p_{y}=p_{z}=0\right)$ the four-dimensional Dirac equation decomposes into two equivalent two-dimensional equations with 2-component spinors and $2 \times 2$ matrices [20]. Then, there results that the scalar and vector interactions preserve their Lorentz structures whereas the anomalous magnetic interaction turns out to be a pseudoscalar interaction. Furthermore, in the $1+1$ world there is no angular momentum so that the spin is absent. Therefore, the $1+1$ dimensional Dirac equation allow us to explore the physical consequences of the negative-energy states in a mathematically simpler and more physically transparent way.

In the presence of a time-independent scalar potential the $1+1$ dimensional time-independent Dirac equation for a fermion of rest mass $m$ reads

$$
\left[c \alpha p+\beta\left(m c^{2}+V\right)\right] \Psi=E \Psi
$$

where $E$ is the energy of the fermion, $c$ is the velocity of light and $p$ is the momentum operator. $\alpha$ and $\beta$ are Hermitian square matrices satisfying the relations $\alpha^{2}=\beta^{2}=1,\{\alpha, \beta\}=0$. From the last two relations it follows that both $\alpha$ and $\beta$ are traceless and have eigenvalues equal to \pm 1 , so that one can conclude that $\alpha$ and $\beta$ are even-dimensional matrices. One can choose the $2 \times 2$ Pauli matrices satisfying the same algebra as $\alpha$ and $\beta$, resulting in a 2-component spinor $\Psi$. The positive definite function $|\Psi|^{2}=\Psi^{\dagger} \Psi$, satisfying a continuity equation, is interpreted as a probability position density and its norm is a constant of motion. This interpretation is completely satisfactory for single-particle states [21]. Using $\alpha=\sigma_{2}, \beta=\sigma_{1}$ and provided that the 
spinor is written in terms of the upper and the lower components

$$
\Psi=\left(\begin{array}{c}
\Psi_{+} \\
\Psi_{-}
\end{array}\right)
$$

the Dirac equation decomposes into :

$$
\begin{aligned}
& E \Psi_{+}=\left(m c^{2}+V\right) \Psi_{-}-\hbar c \Psi_{-}^{\prime} \\
& E \Psi_{-}=\left(m c^{2}+V\right) \Psi_{+}+\hbar c \Psi_{+}^{\prime}
\end{aligned}
$$

where the prime denotes differentiation with respect to $x$. In terms of $\Psi_{+}$and $\Psi_{-}$the spinor is normalized as $\int_{-\infty}^{+\infty} d x\left(\left|\Psi_{+}\right|^{2}+\left|\Psi_{-}\right|^{2}\right)=1$, so that $\Psi_{+}$and $\Psi_{-}$are square integrable functions. It is remarkable that the Dirac equation with a scalar potential is not invariant under $V \rightarrow V+$ const. Therefore, the absolute values of the energy will have physical significance and the freedom to choose a zero-energy will be lost.

The coupling between the upper and the lower components of the Dirac spinor can be formally eliminated for $E \neq 0$ when Eq. (3) is written a pair of second-order differential equations

$$
-\frac{\hbar^{2}}{2 m} \Psi_{ \pm}^{\prime \prime}+V_{e f f}^{ \pm} \Psi_{ \pm}=E_{e f f} \Psi_{ \pm}
$$

where

$$
\begin{aligned}
& E_{\text {eff }}=\frac{E^{2}-m^{2} c^{4}}{2 m c^{2}} \\
& V_{\text {eff }}^{ \pm}=\frac{V^{2}}{2 m c^{2}}+V \mp \frac{\hbar}{2 m c} V^{\prime}
\end{aligned}
$$

These last results tell us that the solution for this class of problem consists in searching for bounded solutions for two Schrödinger equations. It should not be forgotten, though, that the equations for $\Psi_{+}$or $\Psi_{-}$are not indeed independent because the effective eigenvalue $E_{e f f}$, appears in both equations. Therefore, one has to search for bound-state solutions for $V_{e f f}^{+}$and $V_{e f f}^{-}$with a common eigenvalue. The Dirac eigenvalues are obtained by inserting the effective eigenvalues in (5). 
One should realize that the Dirac energy levels are symmetrical about $E=0$ (see, e.g., Refs. [22] and [23]). This conclusion can be obtained directly from (15) as well as from the charge conjugation. Indeed, if $\Psi$ is a solution with energy $E$ then $\sigma_{3} \Psi^{*}$ is also a solution with energy $-E$ for the very same potential. It means that the potential couples to the positiveenergy component of the spinor in the same way it couples to the negativeenergy component. In other words, this sort of potential couples to the mass of the fermion instead of its charge so that there is no atmosphere for the spontaneous production of particle-antiparticle pairs. No matter the intensity and sign of the coupling parameter, the positive- and the negativeenergy solutions never meet. Thus there is no room for transitions from positive- to negative-energy solutions. This all means that Klein's paradox never comes to the scenario.

It is worth to note that the Dirac equation is covariant under $x \rightarrow-x$ if $V(x)$ does not change sign and $\Psi_{ \pm}(-x)=\Psi_{\mp}(x)$ or $\Psi_{ \pm}(-x)=-\Psi_{\mp}(x)$. This is because the parity operator $P=\exp (i \eta) P_{0} \sigma_{1}$, where $\eta$ is a constant phase and $P_{0}$ changes $x$ into $-x$, changes sign of $\alpha$ but not of $\beta$. For an even-parity potential one can notice that $V_{\text {eff }}^{ \pm}(-x)=V_{e f f}^{\mp}(x)$ whereas $E_{\text {eff }}$ remains unchanged.

Now let us consider a scalar potential in the form in the form $V=\mu|x|^{\delta}$, then the effective potential becomes

$$
V_{e f f}^{ \pm}=\frac{\mu^{2}}{2 m c^{2}}|x|^{2 \delta}+\mu|x|^{\delta} \mp \varepsilon(x) \frac{\hbar \mu \delta}{2 m c}|x|^{\delta-1}
$$

where $\varepsilon(x)$ stands for the sign function. In the subsequent survey for the possible existence of bounded solutions we take advantage of the symmetry for $V_{e f f}^{ \pm}$and only consider the positive side of the $x$-axis. When $\delta>0$ the effective potential goes to infinity as $x \rightarrow \infty$ and it is finite at the origin for $\delta \geq 1$ whereas in the range $0<\delta<1$ it has a singularity given by $\mp \hbar \mu \delta / 2 m c|x|^{1-\delta}$, implying in a potential-well structure for $V_{\text {eff }}^{-}\left(V_{e f f}^{+}\right)$when $\mu>0(\mu<0)$ and an attractive potential less singular than $x^{-1}$ for $V_{e f f}^{+}$ $\left(V_{e f f}^{-}\right)$. Therefore, for $\delta>0$ the power potential leads to effective potentials fulfilling the key conditions to furnish discrete spectra. On the other hand, when $\delta<0$ the effective potential vanishes as $x \rightarrow \infty$ and when $\mu>0$ the effective potential $V_{\text {eff }}^{+}$is always repulsive at the origin in such a way that it is repulsive everywhere. Therefore, for $\delta<0$ and $\mu>0$ the power-law potential does not lead to bound-state solutions. For $\delta<0$ and $\mu<0$, though, there is a potential-well structure for $V_{e f f}^{-}$. The same is true for 
$V_{e f f}^{+}$on the condition that $\delta<-1$. For $-1<\delta<0$ and $\mu<0$ there is an attractive potential $V_{e f f}^{+}$with singularity given by $-\hbar|\mu||\delta| / 2 m c|x|^{1+|\delta|}$. For $\delta=-1$ and $\mu<0$ there is also a potential-well structure for $V_{\text {eff }}^{+}$when $\mu<-\hbar c$, and an attractive potential $V_{e f f}^{+}$with singularity at the origin given by $-|\mu| /|x|$ when $\mu=-\hbar c$, and an attractive inverse-square singularity given by $-\hbar|\mu|(1-|\mu| / \hbar c) / 2 m c x^{2}$ when $-\hbar c<\mu<0$. There is no collapse to the center because the potential is not more attractive than $-\hbar^{2} / 8 m x^{2}$ [24]. As a matter of fact, when $\mu=-\hbar c / 2$ the singular potential assumes its critical value. Therefore, for $\delta<0$ and $\mu<0$ the power-law potential also leads to effective potentials fulfilling the key conditions to furnish discrete spectra.

Up to this point we have considered solutions for $E \neq 0$. This supposition has been explicitly assumed to obtain (4)-(6). Nevertheless, one could also ask for possible zero-energy solutions. These zero-mode energies can be obtained directly from the Dirac equation (3). In this case the first-order differential equations are fully uncoupled. Then the upper and lower components of the Dirac spinor for the power-law potential are expressed by

$$
\Psi_{ \pm}=N_{ \pm} F_{ \pm}(x) \exp \left(\mp \frac{m c}{\hbar} x\right)
$$

where

$$
F_{ \pm}(x)=\left\{\begin{array}{cl}
|x|^{\mp \varepsilon(x) \frac{\mu}{\hbar c}} & , \quad \delta=-1 \\
\exp \left(\mp \frac{\mu}{\hbar c} \varepsilon(x) \frac{|x|^{\delta+1}}{\delta+1}\right) & , \text { for } \delta \neq-1
\end{array}\right.
$$

and $N_{ \pm}$is a normalization constant. Normalizable eigenspinors on the positive side of the $x$-axis are given by

$$
\Psi=\left\{\begin{array}{l}
\Psi_{+}\left(\begin{array}{l}
1 \\
0
\end{array}\right), \text { for } \mu>0 \text { and } \delta>-1 \text { or } \mu<0 \text { and } \delta \leq-1 \\
\Psi_{-}\left(\begin{array}{l}
0 \\
1
\end{array}\right), \text { for } \mu<0 \text { and } \delta>-1
\end{array}\right.
$$

Note that the probability position density of the zero-mode spinor has a lonely hump. Note also that the conditions for the existence of bounded solutions for zero-energies has nothing to do with those ones for $E \neq 0$. In the case of $\delta=-1$ another restriction must be added to the top line of (10): $\mu \leq-\hbar c$. This restriction is necessary for obtaining a differentiable spinor 
at the origin and it means that the inversely linear potential must be enough strong to hold a zero-mode solution.

It is surprising to find Dirac eigenspinors with a vanishing lower component in a theory without a nonrelativistic limit. More surprising is to find a vanishing upper component. Both dramatic circumstances make their appearance due to the particular representations of the matrices $\alpha$ and $\beta$ adopted in this paper. It is instructive at this point to consider for a moment a representation where the eigenspinor presents a more familiar behaviour. Let us write the Dirac equation (11) as

$$
\left[c \sigma_{1} p+\sigma_{3}\left(m c^{2}+V\right)\right] \tilde{\psi}=E \tilde{\psi}
$$

The original spinor is related to $\tilde{\psi}$ by the unitary transformation $\psi=U \tilde{\psi}$, where

$$
U=\frac{1}{\sqrt{2}}\left(\begin{array}{ll}
1 & -i \\
1 & +i
\end{array}\right)
$$

so that $\phi=(\tilde{\phi}-i \tilde{\chi}) / \sqrt{2}$ and $\chi=(\tilde{\phi}+i \tilde{\chi}) / \sqrt{2}$. In the nonrelativistic approximation (11) becomes

$$
\begin{gathered}
\tilde{\chi}=\frac{p}{2 m c} \tilde{\phi} \\
\left(-\frac{\hbar^{2}}{2 m} \frac{d^{2}}{d x^{2}}+V\right) \tilde{\phi}=\left(E-m c^{2}\right) \tilde{\phi}
\end{gathered}
$$

Eq. (13) shows that $\tilde{\chi}$ is of order $v / c<<1$ relative to $\tilde{\phi}$ and Eq. (14) shows that $\tilde{\phi}$ obeys the Schrödinger equation with the potential $V$. Now one can see that when one uses the representation where $\alpha=\sigma_{2}, \beta=\sigma_{1}$ (that one used in this paper) one obtains upper and lower components approximately equal to each other in the nonrelativistic limit. On the other side, in the ultrarelativistic limit one expects that $\tilde{\chi}$ presents a contribution comparable to $\tilde{\phi}$, thus the possibilities $\tilde{\phi} \approx i \tilde{\chi}$ and $\tilde{\phi} \approx-i \tilde{\chi}$ imply into $\phi \approx 0$ and $\chi \approx 0$, respectively. Therefore, one can conclude that the zero-mode solutions given by (10) correspond to the ultrarelativistic limit of the theory.

Now let us focus our attention on a scalar power-law potential in the form

$$
V=-\frac{\hbar c q}{|x|}
$$


where $q$ is a real parameter. Then the effective potential becomes the Kratzer potential

$$
V_{e f f}^{ \pm}=-\frac{\hbar c q}{|x|}+\frac{A_{ \pm}}{x^{2}}
$$

where

$$
A_{ \pm}=\frac{\hbar^{2} q}{2 m}[q \mp \varepsilon(x)]
$$

As seen in the preceding paragraph this potential is able to bind fermions on the condition that $q>0$. The effective Kratzer potential is plotted in Fig. 1 for some illustrative values of $q$. It follows that $E_{\text {eff }}<0$, corresponding to Dirac eigenvalues in the range $-m c^{2}<E<+m c^{2}$. The Schrödinger equation with the Kratzer potential is an exactly solvable problem and its solution, for a repulsive inverse-square term in the potential $\left(A_{ \pm}>0\right)$, can be found on textbooks [24-26. Since we need solutions involving a repulsive as well as an attractive inverse-square term in the potential, the calculation including this generalization is presented.

Defining the dimensionless quantities $\xi$ and $C$,

$$
\xi=\frac{2}{\hbar} \sqrt{-2 m E_{\text {eff }}}|x| \quad \text { and } \quad C=q \sqrt{-\frac{m c^{2}}{2 E_{\text {eff }}}}
$$

and using (41)-(15) and (16) one obtains the equation

$$
\Psi_{ \pm}^{\prime \prime}+\left(-\frac{1}{4}+\frac{C}{\xi}-\frac{2 m A_{ \pm}}{\hbar^{2} \xi^{2}}\right) \Psi_{ \pm}=0
$$

Now the prime denotes differentiation with respect to $\xi$. The normalizable asymptotic form of the solution as $\xi \rightarrow \infty$ is $e^{-\xi / 2}$. As $\xi \rightarrow 0$, when the term $1 / x^{2}$ dominates, the regular solution behaves as $\xi^{s}$, where $s$ is a nonnegative solution of the algebraic equation

$$
s(s-1)-\frac{2 m A_{ \pm}}{\hbar^{2}}=0
$$

viz.

$$
s=\frac{1}{2}\left(1 \pm \sqrt{1+\frac{8 m A_{ \pm}}{\hbar^{2}}}\right) \geq 0
$$

If $A_{ \pm}>0$ there is just one possible value for $s$ (that one with the plus sign in front of the radical) and the same is true for $A_{ \pm}=A_{c}=-\hbar^{2} / 8 m$ when $s=$ 
$1 / 2$, but for $A_{c}<A_{ \pm}<0$ there are two possible values for $s$ in the interval $0<s<1$. If the inverse-square potential is absent $\left(A_{ \pm}=0\right)$ then $s=0$ or $s=1$. The solution for all $\xi$ can be expressed as $\Psi_{ \pm}(\xi)=\xi^{s} e^{-\xi / 2} w(\xi)$, where $w$ is solution of the confluent hypergeometric equation [27]

$$
\xi w^{\prime \prime}+(b-\xi) w^{\prime}-a w=0
$$

with

$$
\begin{aligned}
& a=\frac{b}{2}-C \\
& b=2 s
\end{aligned}
$$

Then $w$ is expressed as ${ }_{1} F_{1}(a, b, \xi)$ and in order to furnish normalizable $\psi_{ \pm}$, the confluent hypergeometric function must be a polynomial. This demands that $a=-N$, where $N$ is a nonnegative integer in such a way that ${ }_{1} F_{1}(a, b, \xi)$ is proportional to the associated Laguerre polynomial $L_{N}^{b-1}(\xi)$, a polynomial of degree $N$. This requirement, combined with the top line of (23), also implies into quantized effective eigenvalues:

$$
E_{\text {eff }}=-\frac{q^{2}}{2(s+N)^{2}} m c^{2}, \quad N=0,1,2, \ldots
$$

with eigenfunctions given by

$$
\Psi_{ \pm}(\xi)=N_{ \pm} \xi^{s} e^{-\xi / 2} L_{N}^{2 s-1}(\xi), \quad s>0
$$

where the new constraint over $s$ is a consequence of the definition of associated Laguerre polynomials. Note that the behavior of $\Psi_{ \pm}$at very small $\xi$ implies into the Dirichlet boundary condition $\left(\Psi_{ \pm}(0)=0\right)$. This boundary condition is essential whenever $A_{ \pm} \neq 0$, nevertheless it also develops for $A_{ \pm}=0$.

The necessary conditions for binding fermions in the Dirac equation with the effective Kratzer potential have been put forward. The formal analytical solutions have also been obtained. Now we move on to consider a survey for distinct cases in order to match the common effective eigenvalue. As we will see this survey leads to additional restrictions on the solutions, including 
constraints involving the nodal structure of the upper and lower components of the Dirac spinor.

When $q=1 / 2$ one has $A_{-}>0$ with $s=3 / 2$, and $A_{+}=A_{c}<0$ with $s=1 / 2$. For $q=1, A_{-}>0$ with $s=2$, and $A_{+}=0$ with $s=1$. For $q>1$ one has $s=q+(1 \mp 1) / 2$, corresponding to $A_{ \pm}>0$. For $0<q<1$ and $q \neq 1 / 2$, though, one has $A_{-}>0$ with $s=q+1$ and $A_{c}<A_{+}<0$ with $s=q$ or $s=-q+1$. The preceding analysis shows that in all the circumstances at least one of the effective potentials has a well structure and the highest well $\left(V_{e f f}^{-}\right)$governs the value of the zero-point energy. Note that $s$ is equal to $q$ for $V_{e f f}^{+}$, and $q+1$ for $V_{e f f}^{-}$, with the additional possibility of $s=-q+1$ for $V_{\text {eff }}^{+}$when $0<q<1$. Therefore, the demand for a common effective eigenvalue implies that $n_{-}=n-1$, where $n=n_{+}$is related to $\Psi_{+}$and $n_{-}$ is related to $\Psi_{-}$. The extra value for $s$ when $0<q<1$ (for $V_{e f f}^{+}$) shows to be an unacceptable solution because it does not provide an integer value for $n_{+}-n_{-}$. The solutions, including the zero-mode ones, can now be written as

$$
\begin{gathered}
\Psi_{+}=N_{+} \xi^{q} e^{-\xi / 2} L_{n}^{2 q-1}(\xi), \quad(q \geq 1 \text { for } n=0) \\
\Psi_{-}=N_{-} \xi^{q+1} e^{-\xi / 2} L_{n-1}^{2 q+1}(\xi) \\
E= \pm m c^{2} \sqrt{1-\left(\frac{q}{q+n}\right)^{2}}, \quad n=0,1,2, \ldots
\end{gathered}
$$

where we have used $L_{0}^{k}(\xi)=1$ and $L_{-1}^{k}(\xi)=0$ for all $k$. Note that $E \approx m c^{2}$ and $E_{\text {eff }} \approx E-m c^{2}$, is valid as long as $n \gg q$, thus the nonrelativistic limit of the theory would be, in a limited sense, a regime of large quantum numbers. On the other hand, in the regime of strong coupling, i.e., for $q \gg 1$, one has $E_{e f f} \approx-m c^{2} / 2+n m c^{2} / q$ and as the coupling becomes extremely strong the lowest effective eigenvalues end up close to $-m c^{2} / 2$, corresponding to Dirac eigenvalues $E$, near zero. Now one sees clearly that the eigenvalues as well as the eigenfunctions for a zero-energy solution, in contrast to what is declared 
in Ref. [23], can be obtained as a limit case of a nonzero-energy solution. Fig. 2 shows the behavior of the positive-eigenenergies as a function of $q$.

The effective Kratzer potential leads to bounded solutions in the range $-m c^{2} / 18 \leq E_{e f f}<0$ when a critical potential $\left(V_{e f f}^{+}\right)$is there and $V_{e f f}^{-}$has a minimum value equal to $-m c^{2} / 6$. For $q=1$ the minimum value of $V_{e f f}^{-}$is $-m c^{2} / 4$ and the effective eigenvalue is greater than or equal to $-m c^{2} / 8$. For $q>1$ the effective potential $V_{e f f}^{ \pm}$has the minimum value $-m c^{2} q / 2(q \mp 1)$ and the effective eigenvalues are greater than or equal to $-m c^{2} q^{2} / 2(q+1)^{2}$. In this last case the fermion tends to avoid the origin more and more as $q$ increases due to higher and higher centrifugal barrier $\left[\hbar^{2} q(q \mp 1) / 2 m x^{2}\right]$, which in turn thrust the minimum of the effective potential further (this shifting is proportional to $(q \mp 1))$ and higher (lower) for $V_{e f f}^{+}\left(V_{e f f}^{-}\right)$in such away that $V_{e f f}^{ \pm} \rightarrow V_{e f f}^{\mp}$.

Fig. 3 illustrate the behavior of the probability position density, $|\Psi|^{2}=$ $\left|\Psi_{+}\right|^{2}+\left|\Psi_{-}\right|^{2}$, for the zero-mode solution for $q=1$. Figs. 4, 5] and 6 illustrate the behavior of the upper and lower components of the Dirac spinor, $\left|\Psi_{+}\right|^{2}$ and $\left|\Psi_{-}\right|^{2}$, and the probability position density, $|\Psi|^{2}=\left|\Psi_{+}\right|^{2}+\left|\Psi_{-}\right|^{2}$, for the positive-energy solutions of the first-, second- and third-excited states for $q=1$. The results for negative energies are the same as far as the charge conjugation does $\Psi_{+} \rightarrow \Psi_{+}^{*}$ and $\Psi_{-} \rightarrow-\Psi_{-}^{*}$ The relative normalization of $\Psi_{+}$and $\Psi_{-}$is obtained by substituting the solutions directly into the original first-order coupled equations (3). The result is not pretty for $E \neq 0$ and we preferred to do it by numerical computation with a symbolic algebra program. Comparison of these figures shows clearly that, except for the zero-eigenmode $(n=0),\left|\Psi_{-}\right|$is comparable in importance to $\left|\Psi_{+}\right|$and $\left|\Psi_{-}\right| \rightarrow\left|\Psi_{+}\right|$as $n$ increases, except for a minor difference near the origin. It should not be forgotten, though, that we have restricted our discussion to the positive half line. For $x \leq 0$ the roles of $\Psi_{+}$and $\Psi_{-}$are reversed.

In conclusion, we have succeed in searching for Dirac bounded solutions for the scalar potential $V=-\hbar c q /|x|$. The satisfactory completion of this task for nonzero eigenenergies has been alleviated by the methodology of effective potentials which has transmuted the question into Sturm-Liouville problems with effective inversely linear plus inversely quadratic potentials for both components of the Dirac spinor. A discrete and nondegenerate spectrum has been found. For a strong enough potential a nondegenerate zero-eigenmode corresponding to the ground state comes to the scene. Only in the regime of extremely strong coupling there are degenerate solutions 
related to the zero-energy solutions, one of the eigenspinor representing the fermion and the other representing the antifermion. Beyond its intrinsic importance as a new solution for a fundamental equation in physics, the problem analyzed in this paper presents unusual results.

\section{Acknowledgments}

The author wishes to thank M.B. Hott for useful discussions and for a critical reading of the paper. This work was supported in part by means of funds provided by CNPq and FAPESP. 


\section{References}

[1] S. Coleman, R. Jackiw, L. Susskind, Ann. Phys. (N.Y.) 93 (1975) 267.

[2] S. Coleman, Ann. Phys. (N.Y.) 101 (1976) 239.

[3] J. Fröhlich, E. Seiler, Helv. Phys. Acta 49 (1976) 889.

[4] A.Z. Capri, R. Ferrari, Can. J. Phys. 63 (1985) 1029.

[5] H. Galić, Am. J. Phys. 56 (1988) 312.

[6] G. t’ Hooft, Nucl. Phys. B 75 (1974) 461.

[7] J. Kogut, L. Susskind, Phys. Rev. D 9 (1974) 3501.

[8] R.S. Bhalerao, B. Ram, Am. J. Phys. 69 (2001) 817.

[9] A.S. de Castro, Am. J. Phys. 70 (2002) 450.

[10] R.M. Cavalcanti, Am. J. Phys. 70(2002) 451.

[11] J.R. Hiller, Am. J. Phys. 70 (2002) 522.

[12] A.S. de Castro, Phys. Lett. A 305 (2002) 100.

[13] Y. Nogami, F.M. Toyama, W. van Dijk, Am. J. Phys. 71 (2003) 950.

[14] H.N. Spector, J. Lee, Am. J. Phys. 53 (1985) 248.

[15] R.E. Moss, Am. J. Phys. 55 (1987) 397.

[16] C.-L. Ho, V.R. Khalilov, Phys. Rev. D 63 (2001) 027701.

[17] A. Kratzer, Z. Phys. 3 (1920) 289.

[18] F. Cooper, A. Khare, R. Musto, A. Wipf, Ann. Phys. (N.Y.) 187 (1988) 1.

[19] Y. Nogami, F.M. Toyama, Phys. Rev. A 47 (1993) 1708.

[20] P. Strange, Relativistic Quantum Mechanics, Cambridge University Press, Cambridge, 1998. 
[21] B. Thaller, The Dirac Equation, Springer-Verlag, Berlin, 1992.

[22] F.A.B. Coutinho, Y. Nogami, Phys. Lett. A 124 (1987) 211.

[23] F.A.B. Coutinho, Y. Nogami, F.M. Toyama, Am. J. Phys. 56 (1988) 904.

[24] L.D. Landau, E.M. Lifshitz, Quantum Mechanics, Pergamon, N. York, 1958.

[25] V.G. Bagrov, D.M. Gitman, Exact Solutions of Relativistic Wave Equations, Kluer, Dordrecht, 1990.

[26] S. Flügge, Practical Quantum Mechanics, Springer-Verlag, Berlin, 1999.

[27] M. Abramowitz, I.A. Stegun, Handbook of Mathematical Functions, Dover, Toronto, 1965. 


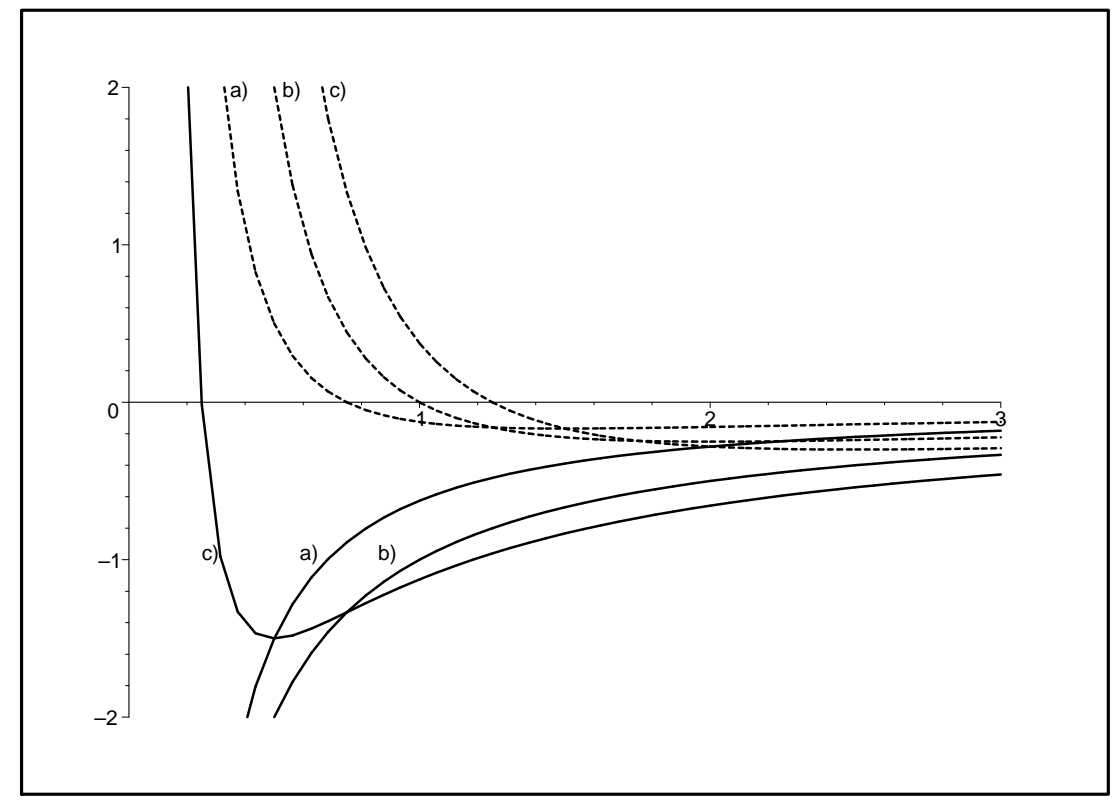

Figure 1: Effective Kratzer potentials as functions of $x$. The solid lines are for $V_{e f f}^{+}$, the dashed lines are for $V_{\text {eff }}^{-}$. $q=3 / 2 \quad(m=\hbar=c=1)$.

a) $q=1 / 2$; b) $q=1$; 


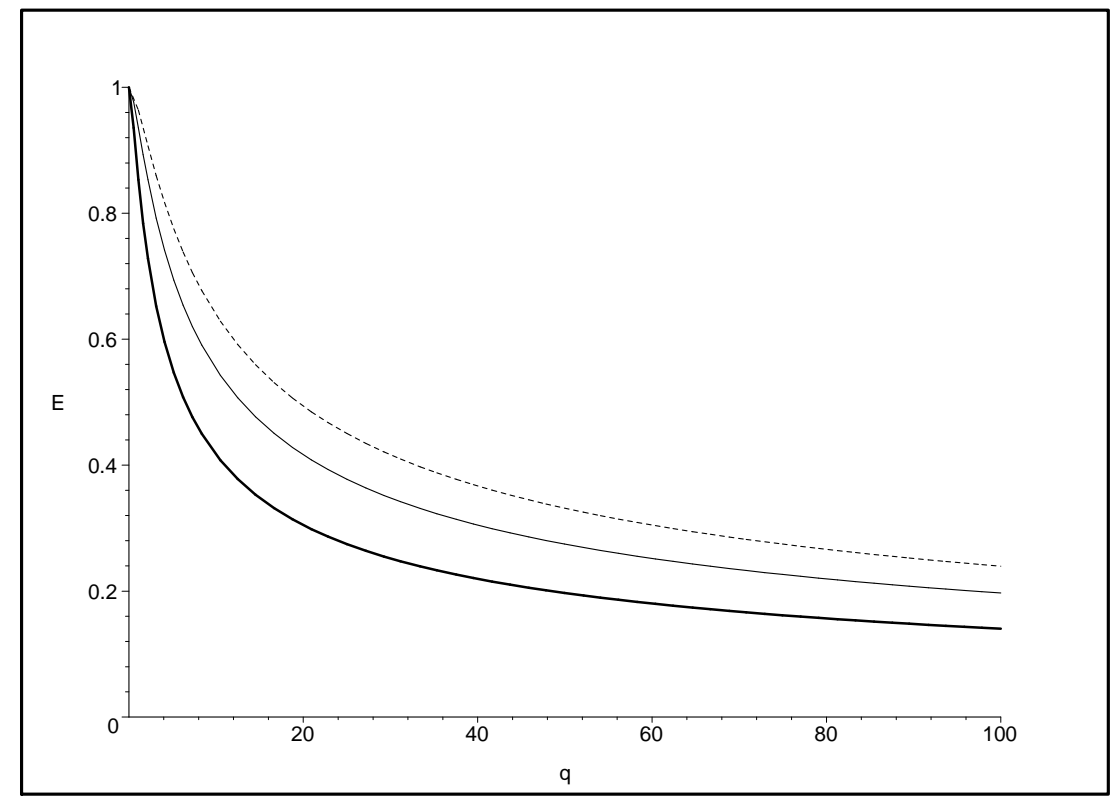

Figure 2: The three lowest positive eigenvalues $(E)$ as a function of $q$ for the potential $V=-\hbar c q /|x|(m=c=1)$. The full thick line stands for $n=1$, the full thin line for $n=2$ and the dashed line for $n=3$. 


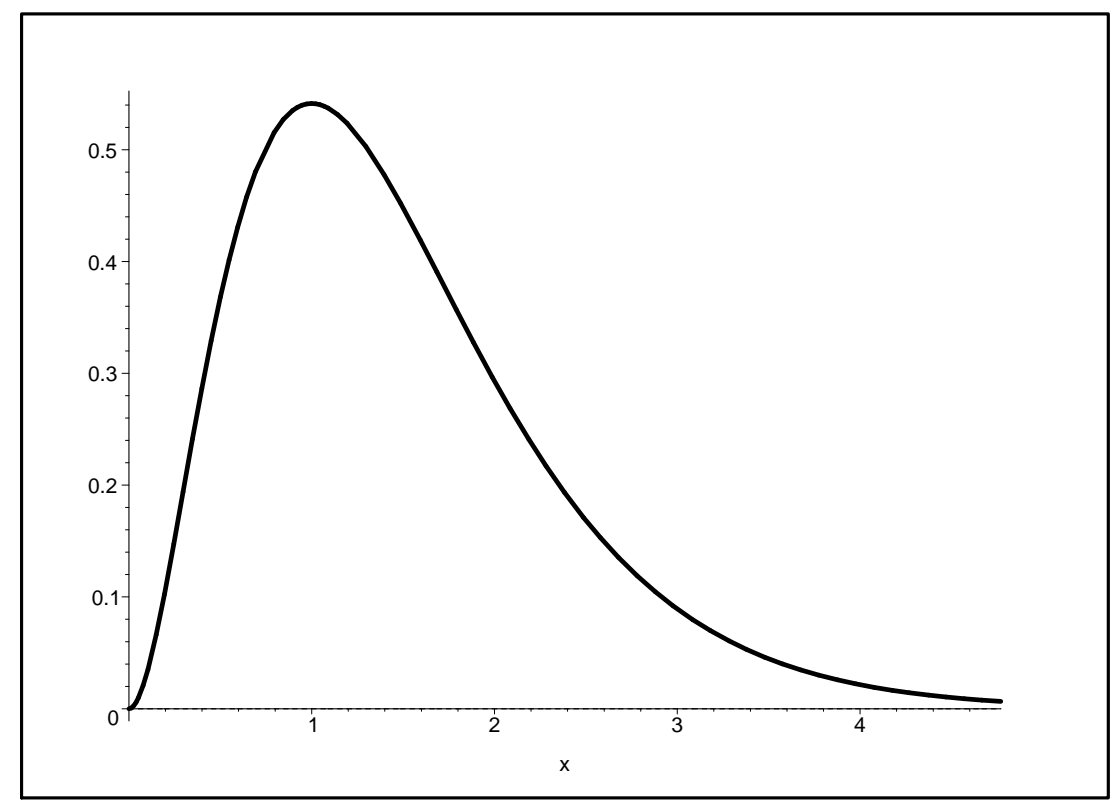

Figure 3: $|\Psi|^{2}=\left|\Psi_{+}\right|^{2}+\left|\Psi_{-}\right|^{2}$ as a function of $x$ corresponding to the ground state $(E=0)$ for the potential $V=-\hbar c q /|x|$ with $q=1(m=c=\hbar=1)$. 


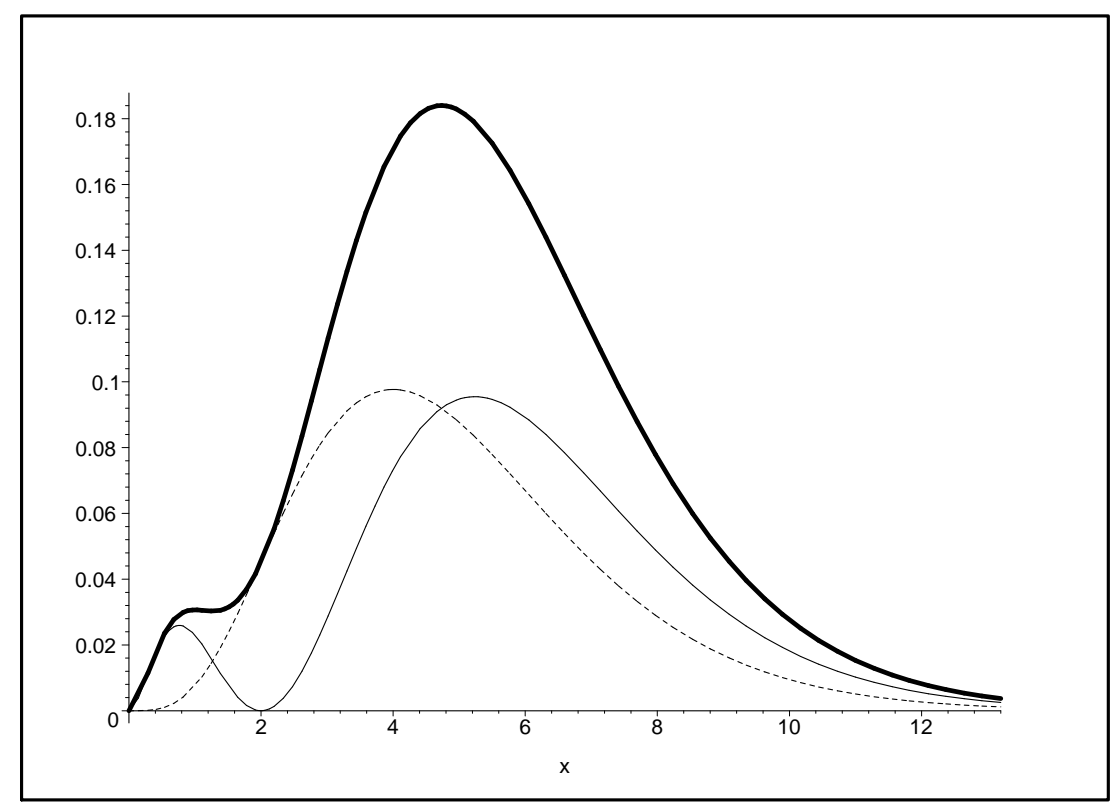

Figure 4: $\left|\Psi_{+}\right|^{2}$ (full thin line), $\left|\Psi_{-}\right|^{2}$ (dashed line) and $|\Psi|^{2}=\left|\Psi_{+}\right|^{2}+\left|\Psi_{-}\right|^{2}$ (full thick line) as a function of $x$, corresponding to the first-excited state $(E>0)$ for the potential $V=-\hbar c q /|x|$ with $q=1(m=c=\hbar=1)$. 


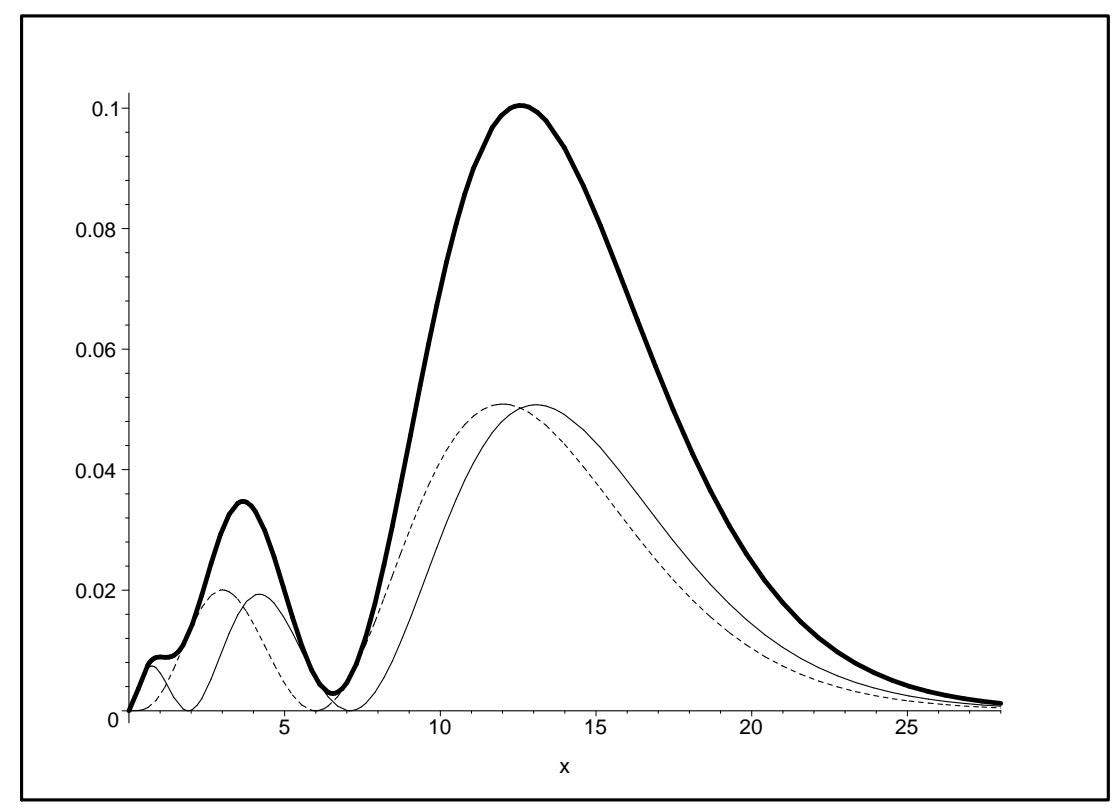

Figure 5: $\left|\Psi_{+}\right|^{2}$ (full thin line), $\left|\Psi_{-}\right|^{2}$ (dashed line) and $|\Psi|^{2}=\left|\Psi_{+}\right|^{2}+\left|\Psi_{-}\right|^{2}$ (full thick line) as a function of $x$, corresponding to the second-excited state $(E>0)$ for the potential $V=-\hbar c q /|x|$ with $q=1(m=c=\hbar=1)$. 


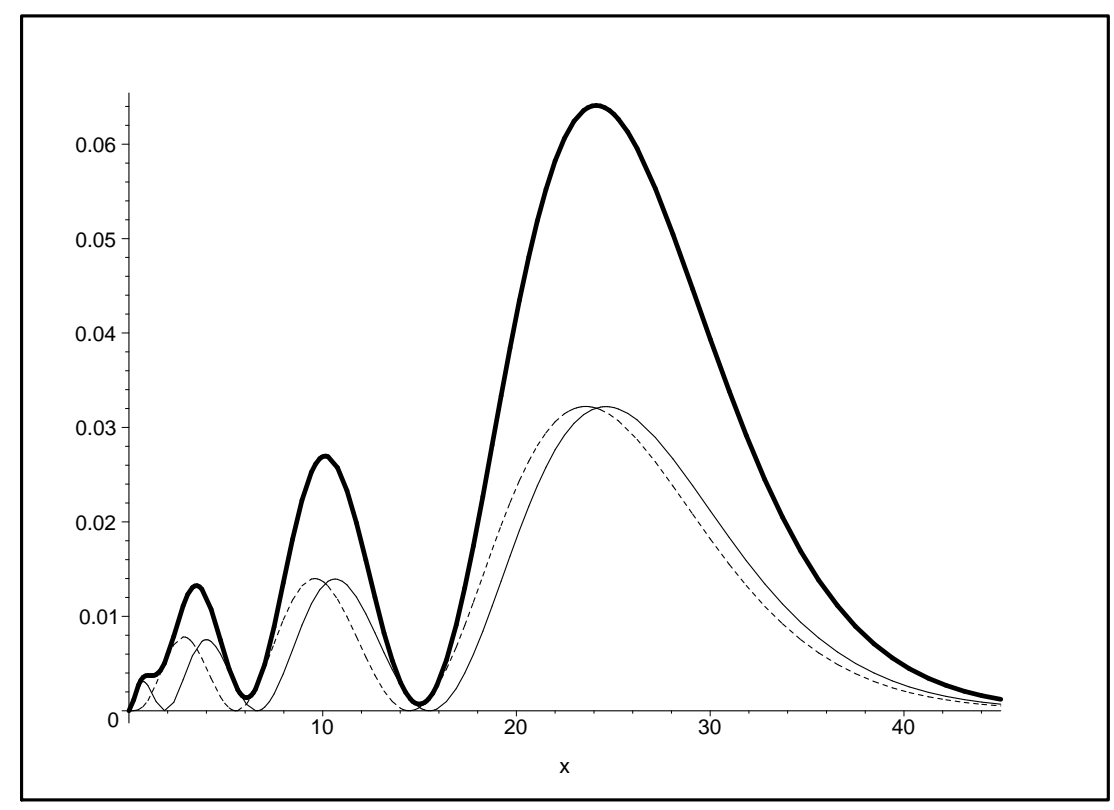

Figure 6: $\left|\Psi_{+}\right|^{2}$ (full thin line), $\left|\Psi_{-}\right|^{2}$ (dashed line) and $|\Psi|^{2}=\left|\Psi_{+}\right|^{2}+\left|\Psi_{-}\right|^{2}$ (full thick line) as a function of $x$, corresponding to the third-excited state $(E>0)$ for the potential $V=-\hbar c q /|x|$ with $q=1(m=c=\hbar=1)$. 